\title{
An Empirical Analysis of Neurofeedback using PID Control Systems
}

\author{
Frank Zeyda, Gabor Aranyi, Fred Charles and Marc Cavazza* \\ School of Computing \\ Teesside University \\ Middlesbrough, TS1 3BA, U.K. \\ f.zeyda@tees.ac.uk g.aranyi@tees.ac.uk f.charles@tees.ac.uk m.o.cavazza@tees.ac.uk
}

\begin{abstract}
Neurofeedback systems can be modeled as closedloop control systems with negative feedback. However, little work to date has investigated the potential of this representation in gaining a better understanding of the actual dynamics of neurofeedback towards explaining subjects' performance. In this paper, we analyze neurofeedback training data through a PID control model. We first show that PID model fitting can produce curves that are qualitatively aligned to the measured BCI signal. Secondly, we examine how brain activity during neurofeedback can be related to common characteristics of control systems. For this, we formalized a pre-existing neurofeedback EEG experiment using a Simulink ${ }^{\circledR}$ model that captures both the neural activity and the external algorithm that was utilized to generate the feedback signal. We then used a regression model to fit individual trial data to PID coefficients for the control model. Our results suggest that successful trials tend to be associated to higher average values of $K_{i}$, which represents the error-reducing component of the PID controller. It hints that convergence in successful neurofeedback is progressive but complete in approaching the target.
\end{abstract}

Index Terms-neurofeedback, BCI, model fitting, PID, linear control systems, statistical analysis, optimization.

\section{INTRODUCTION}

Neurofeedback (NF) [1] can be defined as the mechanism through which human subjects acquire volitional control over the activation of specific brain regions, generally through a process of operant conditioning. The recent development of Brain-Computer Interfaces (BCIs) has seen a growing interest in NF outside neuroscience and clinical research, because of its potential to support the development of BCIs that can be embedded into a variety of interactive software applications.

Despite the intuitive description of any biofeedback system as a control system [2], the idea that NF could be investigated from a control theory perspective has gained acceptance only recently [3]. In such a setting, the "plant" to be controlled corresponds to the target neural activity that the subject is attempting to establish control of, and the negative feedback loop consists of a sensing device measuring the activity of the target brain region, and a method to process and display it.

Such a control system is somehow unique in that it is a hybrid system - neither fully natural like existing physiological systems described through control theory models [2], nor fully artificial like an engineered control system in which

*contact author various parameters can be analytically fine-tuned. This poses distinctive challenges for its description and modeling, and, in particular, its identification from experimental data.

In this paper, we investigate the potential for EEG-based NF systems to be modeled using Linear and Time-Invariant (LTI) control systems. More specifically, we attempt the posthoc modeling of a pre-existing EEG-based NF system using Proportional-Integral-Derivative (PID) control [4], with several objectives in mind. The first one is to confirm whether observed empirical data for NF are compatible with control systems modeling, thereby confirming common intuitions and the most recent proposal of [3]. The second one is to explore whether the PID control system model would allow us to gain specific insights into individual subject performances, such as differentiating between successful and unsuccessful NF trials, or even characterizing control parameters as subject-specific.

To support the current study, we have used data from a previous experiment in which subjects were asked to control their pre-frontal EEG alpha asymmetry as an input signal as part of an affective BCI [5], [6]. The main history of pre-frontal EEG alpha NF has been in clinical studies of depression [7], but the same variable, corresponding to the affective dimension of approach [8], can be used for affective BCI in a variety of software applications, including computer games and interactive narratives, as demonstrated in [6]. The rationale for using a pre-existing NF experiment is that its design would be free of any influence or preconceptions from a control engineering perspective, whilst providing access to significant empirical data sets to be used for model fitting, simulation and subsequent analysis of the controller.

Pre-frontal asymmetry is generally measured through an asymmetry score in the alpha band, such as the $A_{2}$ score defined by $(F 4-F 3) /(F 4+F 3)$ [9]. This variable is known to have trait properties that characterize a given individual [10]; it is moreover amenable to voluntary control on top of the baseline (trait) value, as it also possesses state properties [11]. The EEG $A_{2}$ signal is notoriously difficult to control, beyond the intrinsic difficulty of NF. This is reflected in the variations of success criteria proposed throughout the literature. For instance, [7] proposes counting the number of times that the $A_{2}$ value would cross a predefined threshold to account for the high instability in the EEG signal. We have opted for a signal 


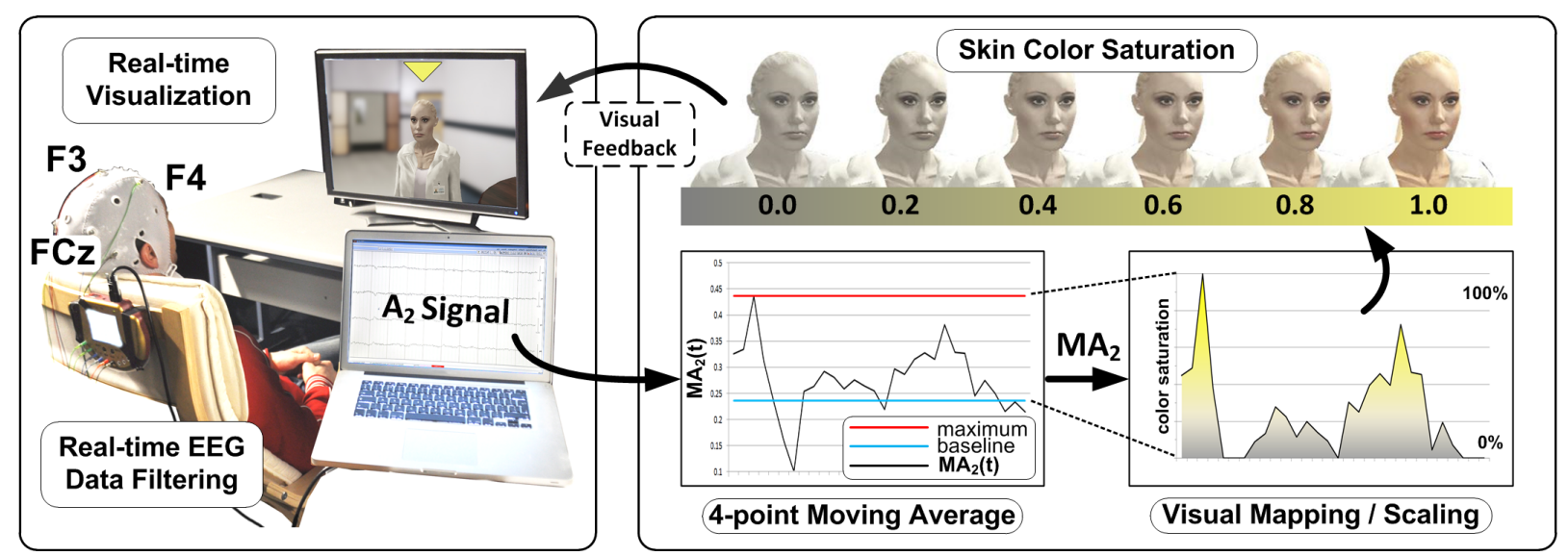

Fig. 1. Experimental setup of our NF installation that was used to collect the empirical data for PID fitting.

more suitable to control systems modeling, using a 4-point moving average for $A_{2}$ and an individual threshold determined by the subject's baseline as suggested in [12], with a success score derived from the integration of above-threshold values over the NF trial epoch. We note that this success criterion is trait dependent, taking the subject's baseline into account.

The use of LTI systems to approximate the behavior of neurofeedback faces similar issues as their use for physiological control systems, in that they are most likely to constitute an approximation of a potentially more complex and non-linear system [2]. It is difficult to hypothesize which components of an NF system actually depart from linearity, because in an NF control model, the "plant" to be controlled artificially groups together two entities: (i) a physiological system corresponding to the target neural activity, and (ii) some internal (not yet fully understood) control mechanism through which subjects gain volitional control of the target neural activity via operant conditioning. Further to linearity, we assume that considering the limited duration of NF epochs, a time-invariant hypothesis is acceptable on all or significant fractions of the NF epoch. It should be noted that we are only interested in NF for real-time BCIs, not the induced effects of NF on the subject, which are most likely to impact on the control model.

In this paper, we therefore consider an NF system as an LTI closed-loop control system with PID negative feedback. Since the objective of NF is to reach a target state and maintain that state for the duration of the NF epoch, we consider the set (target) value as a Heaviside step function, and do not model any explicit perturbation of the target. We provide statistical evidence for PID parameters derived from our model fitting to be significantly correlated with success scores of NF trials.

In Section II, we discuss previous and related work, and Section III explains our experimental setup for the NF. Next, Section IV details the PID control model, and Section V presents a statistical analysis of our findings and fitting results. Lastly, in Section VI we conclude and identify future work.

\section{Previous ANd Related Work}

Héliot et al. [13] have reported the successful use of control theory to model the learning process of an invasive Brain-
Machine Interface in macaque monkeys. Their hypothesis was that closed-loop operation of a brain-machine interface relied on the subject's ability to learn an inverse transformation of the plant (neural activity) to be controlled, and their model was successfully correlated with experimental data.

Ros et al. [3] have provided the most recent and comprehensive proposal for a control systems approach to NF, albeit not necessarily in the context of linear systems. Their central argument is that while specific neural activity is the target of control, it is the feedback channel of NF (its sensing component) that makes the overall system a closed-loop control circuit [3]. They draw upon previous considerations on the applicability of control systems to neuroscience [13], [14]. One difference to our work here is that their interest is mainly in $\mathrm{NF}$ learning (through the neural modifications induced by NF in the subject, such as Hebbian plasticity), and ours in using NF for BCIs, which may set a different context for control systems analysis - in particular, how set-point values are determined.

The use of PID controllers has been reported for automatic adaptation of game level difficulty in biofeedback games [15]. While this approach shares some of the topics addressed in our own work, Parnandi and Gutierrez-Osuna [15] did not use PID controllers to model the biofeedback process itself.

However, one of the most relevant works to date has been a PID model of human balance keeping, introduced by Hidenori and Jiang [16], in which PID coefficients were determined from experimental data on the subjects' balance, with the aim of differentiating between normal and pathological balance control, as encountered in certain medical conditions. We have drawn part of our methodology in terms of PID experimental data fitting from their work [16].

We next review the experimental setup that was used to obtain the NF data employed later on for PID fitting.

\section{EXPERIMENTAL SETUP}

The NF experiments that have provided the data we analyze here have been carried out as part of previous research in affective BCI [17]. In these experiments, we explored the ability of subjects to control their level of pre-frontal EEG 


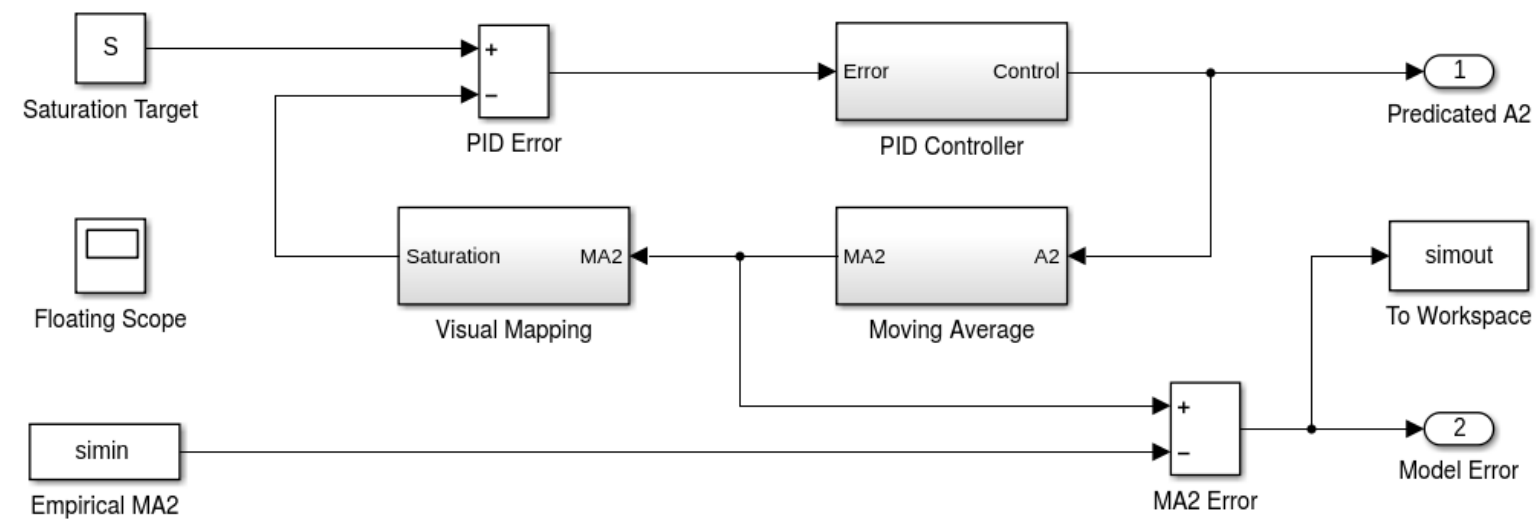

Fig. 2. Simulink ${ }^{\circledR}$ control model of the NF experimental setup depicted in Fig. 1.

alpha asymmetry as a marker of approach, as proposed in [8]. The cognitive task that subjects were given as part of the experiment was to mentally support a virtual character, which is expected to result in an increase of left pre-frontal asymmetry.

The overall setting is depicted on Fig. 1. Subjects were equipped with an EEG cap from which alpha band $(8-12 \mathrm{~Hz})$ power was extracted online from the electrodes $F 3$ and $F 4$, sampled at $1 \mathrm{~Hz}$ with a reference electrode at $F C z$. The 4point moving average $M A_{2}$ of the $A_{2}$ asymmetry score was computed in real-time as input to the system. The feedback channel consisted in the color saturation level of a virtual character: the more the subjects were perceived to support the character (through variation of their $M A 2$ signal), the more the character restored to its original skin hue. Each subject went through $12 \mathrm{NF}$ training epochs of 32 seconds, each preceded by a 15 seconds resting period. Because the baseline value of $A_{2}$ acts as a characteristic feature of each individual, success scores had to be defined individually, after an initial calibration step in which $A_{2}$ was measured (eyes open) for 120 seconds. During NF, the real-time $M A_{2}$ value is mapped onto color saturation in a linear fashion to yield a percentage with $0 \%$ corresponding to the subject's baseline and $100 \%$ to the maximum variation of $A_{2}$ observed across subjects during calibration (empirically determined as baseline +0.2 ). Success over a given NF block is determined by calculating the average saturation value over the 32 seconds block, with a success threshold of $10 \%$, which is equivalent to approximately $50 \%$ saturation over 6 seconds. As a caveat, for successful epochs, we also require that the score has to increase with respect to the average of the preceding resting period.

\section{NeurofeEdBACK CONTROL Model}

We have used the Simulink ${ }^{\circledR}$ [18] software to facilitate modeling and simulation of our control system. Simulink ${ }^{\circledR}$ integrates with the MATLAB ${ }^{\circledR}$ platform, which provides a rich set of functions to perform mathematical operations on vectors and matrices. Notable features of Simulink ${ }^{\circledR}$ are support for both discrete and continuous models, and the ability to extract and visualize data from simulations via program scripts.
Fig. 2 illustrates the Simulink ${ }^{\circledR}$ control model of our NF experimental setup described above. We use a PID Controller to model the subject's cognitive process for the NF. The output of the PID (Control) corresponds to the $A_{2}$ signal predicated by the model, and the PID input (Error) is the error between the saturation (visual feedback) and a fixed target $S$ for the saturation score, whose empirical definition corresponds to the NF task. The visual feedback is calculated from the $A_{2}$ signal by virtue of two subsystems, one for the 4-point Moving Average and another for the subject-specific Visual Mapping, mirroring our setup in Fig. 1. The model, in addition, includes a source block for the empirical $M A_{2}$ signal, which is used to calculate the error between empirical and control model $M A_{2}$.

For brevity, we omit a detailed description of the subsystems PID Controller, Moving Average, and Visual Mapping, as their definition is standard [19]. For the Moving Average block, we use the difference between two integrators, one of them being delayed by 4 time steps; the Visual Mapping block implements the arithmetic operations to map signal values from the interval [baseline, $\min (0.7,0.2+$ baseline $)]$ into the interval $[0,1]$, where baseline is the subject's $A_{2}$ baseline. A detailed justification of the mapping is in [17]. We have both, a discrete and continuous version of the Simulink ${ }^{\circledR}$ model. For simulation, we shall use the continuous version.

The Saturation Target block outputs a Heaviside function scaled by the constant $S$. The use of a Heaviside function as our target reflects the assumption noted earlier that subjects are trying to maximize the visual feedback during the entire 32 seconds trial period. We have set the saturation target to $100 \%$, even though subjects may not be able to sustain their maximum asymmetric activity for a prolonged period of time ${ }^{1}$. However, experimental results show that a target of $100 \%$ does indeed yield the best approximation of the control model $M A_{2}$ vs the empirical $M A_{2}$ signal; while in some cases, a local optimum for $S$ near $60 \%$ can be observed, the performance improvement of the PID fitting for values of $S$ above $70 \%$ turns out to be only marginal in the majority of cases.

\footnotetext{
${ }^{1}$ Due to the individualized definition of saturation, a $100 \%$ saturation target does not correspond to an asymmetry score of 1.0 , which would actually be unrealistic to achieve.
} 


\section{A. PID Fitting}

The control behavior of a PID is typically characterized by three constants: $K_{p}, K_{i}$ and $K_{d}$. They are weights (gains) that are respectively applied to the current value of the control error, integral of the error, and derivative of the error. The sum of the three weighted signals determines the actual control output of the PID - here being the $A_{2}$ signal. To derive the gains for a given subject and trial epoch, we use the empirically measured $A 2$ signal and visual feedback (saturation signal) obtained from the experimental data reported in [17]. We have $A_{2}$ signal data for 22 subjects, each providing 12 trial epochs; we recall that a trial epoch samples $A_{2}$ over a 32 seconds window, at a rate of $1 \mathrm{~Hz}$. Fitting is done on a per-trial basis.

The derivation of optimal PID gains is achieved by solving a least-mean-squares (LMS) optimization problem in the time domain. Generally, the output $c(t)$ of a continuous-time ${ }^{2}$ PID with input $e(t)$ is determined by the following equation.

$$
c(t)=K_{p} e(t)+K_{i} \int_{0}^{t} e(t) d t+K_{d} \frac{d e}{d t}
$$

In our case, $c(t)$ corresponds to the measured $A_{2}$ signal and $e(t)$ can be calculated from it via the 4-point moving average and visual mapping functions realized by the blocks in Fig. 2. Furthermore, for a given $e(t)$ signal, we can calculate a priori its integral $e_{i}(t)$ and derivative $e_{d}(t)$. Equation 1 then gives rise to a system of 32 linear equations - one for each sample. It can be more concisely written in matrix form as follows.

$$
c=E K
$$

where

$$
E=\left(\begin{array}{ccc}
e(1) & e_{i}(1) & e_{d}(1) \\
e(2) & e_{i}(2) & e_{d}(2) \\
\vdots & \vdots & \vdots \\
e(32) & e_{i}(32) & e_{d}(32)
\end{array}\right) \text { and } K=\left(\begin{array}{c}
K_{p} \\
K_{i} \\
K_{d}
\end{array}\right)
$$

The system is over-determined and thus generally does not have an exact solution; but we can use LMS regression to find a vector $K$ that best approximates the left-hand and righthand side of (2). Formally, this is minimizing the residual $|r|^{2}$ in $c=E K+r$. Generally, we obtain $K=\left(E^{T} E\right)^{-1} E^{T}$ as the unique solution to this linear optimization problem.

In practical terms, for model fitting we ignore the first input tuple $\left(e(1), e_{i}(1), e_{d}(1)\right)$ since the differential error $e_{d}(1)$ is not meaningful as the first measurement is taken. Because the control analysis is done in MATLAB ${ }^{\circledR}$, we can take advantage of built-in functions to solve the LMS problem. We thereby obtain a triple $\left(K_{p}, K_{i}, K_{d}\right)$ for each trial of each subject, and those triples provide the basis for further statistical analysis.

We note that the three $K$ values fully characterize the subject's NF process as a PID controller. In real-life control engineering, PIDs often additionally specify a filter coefficient for the derivate action, which we do not consider in our model.

\footnotetext{
${ }^{2}$ While NF data acquisition is usually discrete, any exact control model for NF must be essentially continuous. The choice of a discrete over continuous model only affects simulation but not the PID fitting approach per se.
}
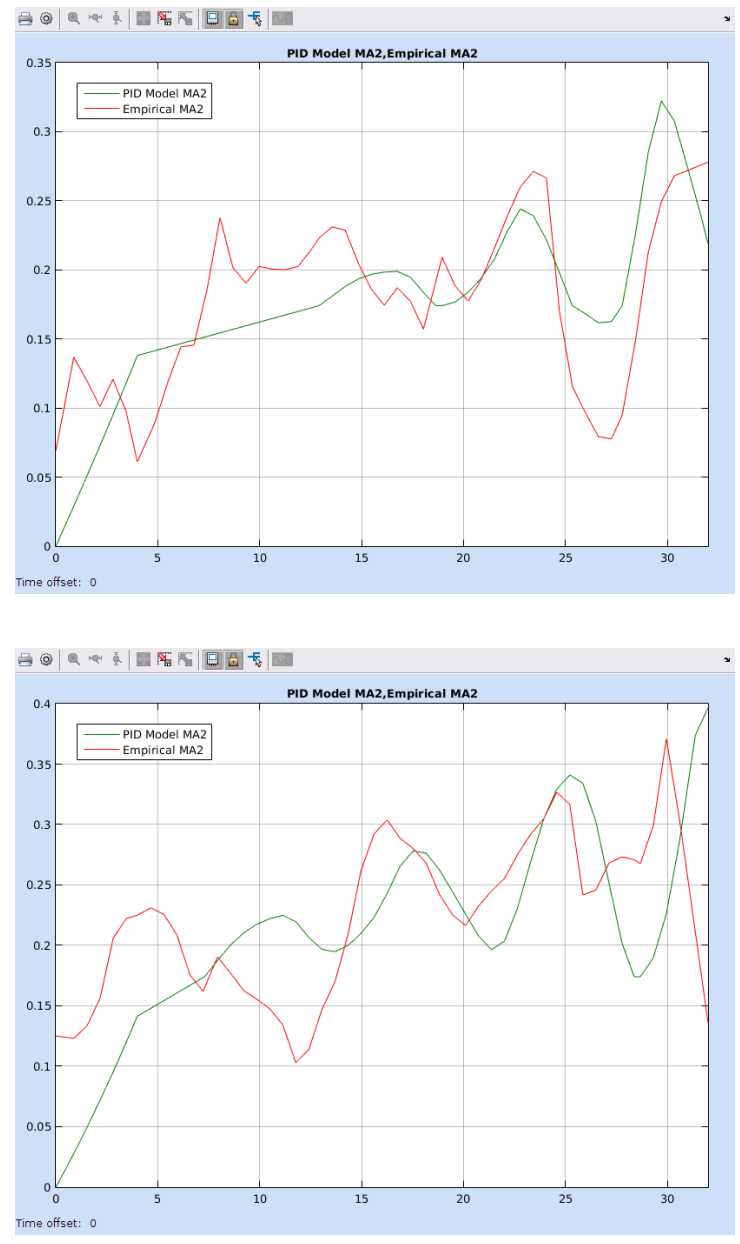

Fig. 3. Signal plots for simulations of the fitted PID control system.

\section{B. Simulation of the Control Model}

To visually confirm the quality of the fitted model, we have used the estimated $K$-gains to simulate the NF control system in Fig. 2. Fig. 3 includes two sample plots of the predicted $M A_{2}$ (green line) vs measured $M A_{2}$ (red line) for two trials of a successful subject (no. 6233, trials $2 \& 6$ ). To validate the simulation, we examine the $M A_{2}$ signals rather than the raw $A_{2}$ signals because $M A_{2}$ already filters out some of the noise and erratic (high-frequency) oscillations present in $A_{2}$.

While the two signals are subjectively well aligned for these particular trials and subject, this is not consistently the case for all trials and subjects. For the majority of the cases, however, we can observe that the trend of the simulated signal is visually aligned with that of the empirical one, though the signal levels may not always peak at comparable magnitudes.

Simulation in Simulink ${ }^{\circledR}$ allows us furthermore to derive a quantitative measure for the quality of the fitting. For this, we calculate the integral of the absolute error between empirical and model $M A_{2}$ over the 32 seconds sample window.

While visual validation generally confirmed viability of the model fitting approach, we next report on a statistical analysis of the $K$-gains in order to examine their correlation to the success and failure behavior of subjects during the NF trials. 


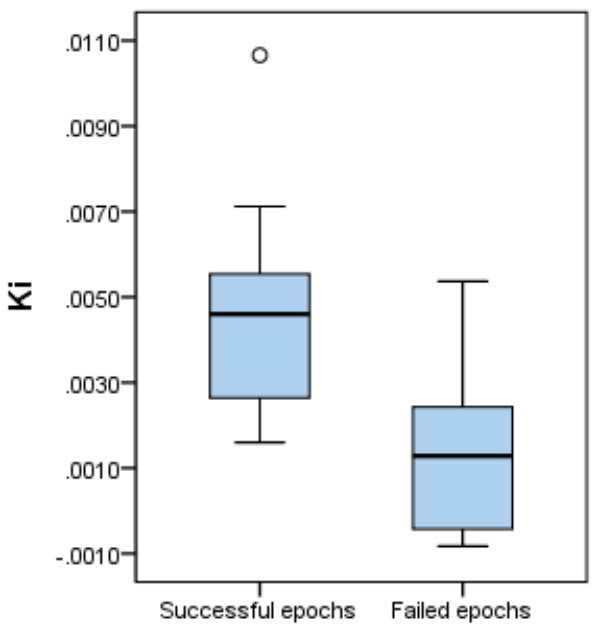

Fig. 4. Boxplot: $K_{i}$ in successful vs failed epochs.

\section{Statistical Analysis}

Our hypothesis is that the parameters of the fitted PID model enable us, with reasonable certainty, to discriminate between successful and failed NF trials. Towards confirming this, we first examined the three $K$-gains across the 12 trial epochs of each subject. For each subject, we calculated the mean and standard deviation (SD) of, $K_{p}, K_{i}$ and $K_{d}$ for successful and unsuccessful epochs, respectively. This revealed that the mean difference of $K_{i}$ between successful and unsuccessful trials of a subject exhibited a discernible pattern: for 13 of the 22 subjects (59\%), the mean of $K_{i}$ [success] was indeed larger than the mean of $K_{i}$ [failure].

We note that data for 5 out of the 22 subjects had to be ignored in this analysis due to those subjects having either no successful or no failed trials. Hence, in $76 \%$ of the permissible trials, the aforementioned pattern could be observed. In addition, for 9 subjects (53\%), the mean difference in $K_{i}$ was centered around $5 \cdot 10^{-3}\left( \pm 2 \cdot 10^{-3}\right)$, whereas $K_{p}$ and $K_{d}$ seem to vary more randomly with no discernible pattern.

A dependent t-test revealed that the mean of $K_{i}$ was significantly larger in successful trials, $M=0.04, S D=3.9 \cdot 10^{-3}$, than failed trials, $M=1.2 \cdot 10^{-3}, S D=1 \cdot 10^{-3}, t(16)=2.66$, $p=0.02$, (large effect-size). This initial analysis suggests that $K_{i}$ may be a good candidate for a predictor of NF success. To further explore this surmise, we considered the correlation of $K_{p}, K_{i}$ and $K_{d}$ with the raw (continuous) NF score. This was done separately for successful and unsuccessful trials across all subjects. Overall, we utilized a pool of 264 trial epochs, of which 110 had successful and 154 had failed NF outcomes.

We observed that in successful trials, the NF score was significantly (positively) correlated with $K_{i}, r=0.32$ for $p<0.001$ (95\% CI for $r=[0.14 ; 0.48]$ ), while there was no significant correlation of $K_{i}$ with the NF score in unsuccessful trials, $r=0.09, p=0.29, n s$. Interestingly, we also discovered that the NF score in unsuccessful trials was significantly (negatively) correlated with $K_{d}, r=-0.36, p<0.001$ (95\% CI for $r=[-0.22 ;-0.50])$, whereas there was no

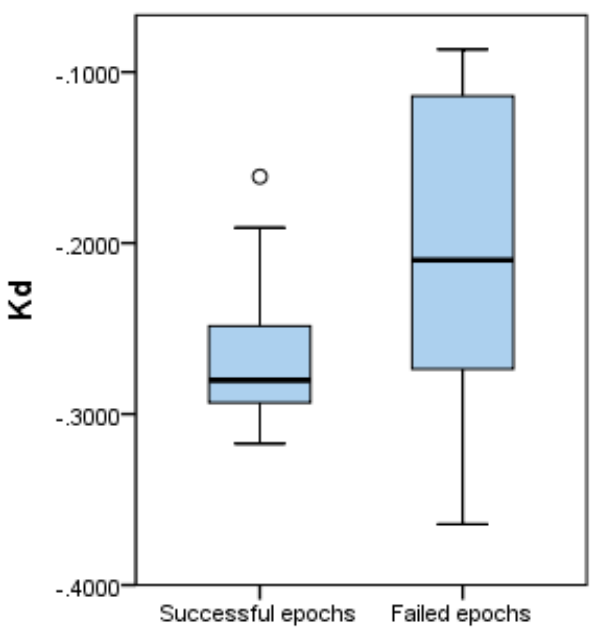

Fig. 5. Boxplot: $K_{d}$ in successful vs failed epochs.

significant correlation between $K_{d}$ and scores in successful trials, $r=-0.17, p=0.08, n s$. This supports the claim that $K_{i}$ indeed acts as an indicator for NF success, whereas $K_{d}$ may be associated with NF failure. Regarding $K_{p}$, we found no correlation with neither successful nor failed trials.

To further explore the relationship between $K$-gains and trial epoch success, we averaged the $K_{i}$ and $K_{d}$ values over successful and failed trials for subjects who had at least 3 successful trials and had at least a single failed trial (13 subjects). This was to exclude subjects with $\mathrm{BCI}$ illiteracy, assuming that they are not amenable to description by our control model. Applying a Wilcoxon signed-rank (non-parametric) test showed that $K_{i}$ was significantly larger in successful epochs $\left(M d n=4.6 \cdot 10^{-3}\right)$ than in failed ones $\left(M d n=1.3 \cdot 10^{-3}\right)$, $T=7, z=-2.69, p=0.01$, and $r=-0.74$ (large effectsize). The same test with $K_{d}$ as dependent variable showed that the difference between $K_{d}$ in failed epochs $(M d n=-0.21)$ and in successful epochs $(M d n=-0.28)$ approached significance, $T=19, z=-1.85, p=0.06, r=0.51$ (large effectsize). Boxplots indicating $K_{i}$ and $K_{d}$ for successful and failed epochs are presented in Figs 4 and 5. The middle line is the median, the bottom and top of the boxes are the $1^{\text {st }}$ and $3^{\text {rd }}$ quartile, and the whiskers indicate lowest and highest values within $2 S D$ from the mean. Only two trials lie outside; they are referred to as outliers and indicated with a circle.

From Fig. 4, we observe that the smallest $K_{i}$ in successful epochs is larger than the median $K_{i}$ in failed epochs, supporting discriminability of success based on $K_{i}$. Conversely, the range of $K_{d}$ in successful epochs (Fig. 5) completely overlaps with the range of $K_{d}$ in failed epochs.

These findings should be interpreted considering the known effects [19] of each PID constant on the closed-loop response, although with some caveats due to the specific conditions of biological systems. $K_{i}$ 's main effect is to eliminate steadystate error, and in that sense it plays a major role in reaching the target NF value. It is thus consistent that an improvement in eliminating steady-state error results in more successful NF, although the neurofeedback epoch is more likely to comprise 
several short bursts of steady-state periods. The interpretation of $K_{d}$ 's impact is more challenging. Its nominal effect is to reduce overshoot and settling time; however, it is also to increase noise sensitivity. Since noise is very likely to be a major issue in EEG neurofeedback, our tentative explanation is that noise sensitivity should constitute the main impact of $K_{d}$ on NF, and this impact would actually be detrimental.

\section{CONCLUSION}

We have presented a first attempt at modeling an NF installation as a PID control system. Experimental NF data was used to obtain coefficients for the best fit of the PID controller on a per-trial basis, and we identified a correlation between the values of those coefficients and failure / success of the underlying NF experiments. The significance of our findings is to provide evidence that neurofeedback can be meaningfully described and analyzed through linear control models.

The hybrid nature of NF systems, which contain elements of experimental design, and physiological elements which are not yet fully understood, certainly constitutes a challenge when recurring to traditional systems-modeling techniques pertaining to general control theory. This is why we should emphasize the inherently heuristic nature of our findings. However, formal modeling tools can play an essential role in supporting fundamental efforts in understanding the inner mechanisms of $\mathrm{NF}$ and discover models that approximate them.

In addition to recent proposals based on control theory [3], the endeavor of unravelling NF mechanisms should be traced to the analysis of success determinants such as, for instance, instructions [20] or cognitive strategies [21]. While formalization may help understanding empirical NF results, theoretical discussions on the "nature of control" in NF systems [22] should also play an essential role in improving the type of control systems modeling we have introduced here.

As for future work, we suggest a deeper and quantitative analysis of the prediction error of the PID control model vs the experimental NF data. It would additionally be interesting to determine a possible relationship between the quality of the model fitting and the PID coefficients, with the ultimate goal of showing that they entail subject-specific traits. The baseline of subjects could also be included in this analysis, for instance, by examining whether it affects success of the fitting. Another future work is a detailed examination of stability of the fitted model, using, for instance, Nyquist plots.

Acknowledgment: The original neurofeedback experiments [17] from which we derived data analyzed here have been undertaken in collaboration with the Functional Brain Center of the Tel Aviv Sourasky Medical Center (Prof. Talma Hendler). Ilana Klovatch and Gilan Jackont are thanked for their help with EEG data acquisition during these experiments.

\section{REFERENCES}

[1] C. Neuper and G. Pfurtscheller, "Neurofeedback training for BCI control," in Brain-Computer Interfaces, ser. The Frontiers Collection. Springer, Sep. 2010, pp. 65-78.

[2] M. C. K. Khoo, Physiological Control Systems: Analysis, Simulation, and Estimation. Wiley-IEEE Press, Sep. 1999.
[3] T. Ros, B. J. Baars, R. A. Lanius, and P. Vuilleumier, "Tuning pathological brain oscillations with neurofeedback: A systems neuroscience framework," Frontiers in Human Neuroscience, vol. 8, no. 1008, Dec. 2014.

[4] K. H. Ang, G. Chong, and Y. Li, "PID control system analysis, design, and technology," IEEE Transactions on Control Systems Technology, vol. 13, no. 4, pp. 559-576, Jul. 2005.

[5] S. Gilroy, J. Porteous, F. Charles, M. Cavazza, E. Soreq, G. Raz, L. Ikar, A. Or-Borichov, U. Ben-Arie, I. Klovatch, and T. Hendler, "A braincomputer interface to a plan-based narrative," in Proceedings of the 23rd International Joint Conference on Artificial Intelligence (IJCAI 13). AAAI Press, Aug. 2013, pp. 1997-2005.

[6] S. Gilroy, J. Porteous, F. Charles, M. Cavazza, I. Klovatch, G. Raz, L. Ikar, E. Soreq, U. Ben-Arie, and T. Hendler, "A neurofeedback approach to supporting characters in virtual stories," in Proceedings of the 5th International Brain-Computer Interface Meeting. Graz University of Technology Publishing House, Jul. 2013.

[7] J. P. Rosenfeld, G. Cha, T. Blair, and I. H. Gotlib, "Operant (biofeedback) control of left-right frontal alpha power differences: Potential neurotherapy for affective disorders," Biofeedback and Self-regulation, vol. 20, no. 3, pp. 241-258, Sep. 1995.

[8] R. J. Davidson, P. Ekman, C. D. Saron, J. A. Senulis, and W. V. Friesen, "Approach-withdrawal and cerebral asymmetry: Emotional expression and brain physiology I," Journal of Personality and Social Psychology, vol. 58 , no. 2, pp. 330-341, Feb. 1990.

[9] R. J. Davidson, "EEG measures of cerebral asymmetry: Conceptual and methodological issues," International Journal of Neuroscience, vol. 39, no. $1-2$, pp. 71-89, Mar. 1988.

[10] A. J. Tomarken, R. J. Davidson, R. E. Wheeler, and L. Kinney, "Psychometric properties of resting anterior EEG asymmetry: Temporal stability and internal consistency," Psychophysiology, vol. 29, no. 5, pp. 576-592, Sep. 1992.

[11] J. A. Coan and J. J. B. Allen, "The state and trait nature of frontal EEG asymmetry in emotion," in The Asymmetrical Brain, K. Hugdahl and R. J. Davidson, Eds. MIT Press, 2002, pp. 566-615.

[12] V. Zotev, R. Phillips, H. Yuan, M. Misaki, and J. Bodurka, "Selfregulation of human brain activity using simultaneous real-time fMRI and EEG neurofeedback," Neurolmage, vol. 85, Part 3, no. 0, pp. 985995, Jan. 2014.

[13] R. Héliot, K. Ganguly, J. Jimenez, and J. M. Carmena, "Learning in closed-loop brain-machine interfaces: Modeling and experimental validation," IEEE Transactions on Systems, Man, and Cybernetics, Part $B$, vol. 40, no. 5, pp. 1387-1397, Oct. 2010.

[14] B. Grychtol, H. Lakany, G. Valsan, and B. A. Conway, "Human behavior integration improves classification rates in real-time BCI," IEEE Transactions on Neural Systems and Rehabilitation Engineering, vol. 18, no. 4, pp. 362-368, Aug. 2010.

[15] A. Parnandi and R. Gutierrez-Osuna, "A comparative study of game mechanics and control laws for an adaptive physiological game," Journal on Multimodal User Interfaces, vol. 9, no. 1, pp. 31-42, Mar. 2015.

[16] K. Hidenori and Y. Jiang, "A PID model of human balance keeping," IEEE Control Systems, vol. 26, no. 6, pp. 18-23, Dec. 2006.

[17] M. Cavazza, G. Aranyi, F. Charles, J. Porteous, S. Gilroy, G. Jackont, I. Klovatch, G. Raz, N. J. Keynan, A. Cohen, and T. Hendler, "Frontal alpha asymmetry neurofeedback for brain-computer interfaces," in Proceedings of the 6th International Brain-Computer Interface Conference, Graz, Austria, Dec. 2014.

[18] The MathWorks, Inc., "Simulink: Simulation and model-based design," http://uk.mathworks.com/products/simulink/, 1994-2015.

[19] M. A. Johnson and M. Moradi, "PID control technology," in PID Control: New Identification and Design Methods, M. A. Johnson and M. Moradi, Eds. Springer, 2005, pp. 1-46.

[20] F. Lotte, F. Larrue, and C. Mühl, "Flaws in current human training protocols for spontaneous Brain-Computer Interfaces: lessons learned from instructional design," Frontiers in Human Neuroscience, vol. 7, no. 568, Sep. 2013.

[21] E. V. C. Friedrich, G. Wood, R. Scherer, and C. Neuper, "Mind over brain, brain over mind: cognitive causes and consequences of controlling brain activity," Frontiers in Human Neuroscience, vol. 8, no. 348, May 2014

[22] G. Wood, S. E. Kober, M. Witte, and C. Neuper, "On the need to better specify the concept of "control" in brain-computer-interfaces / neurofeedback research," Frontiers in Systems Neuroscience, vol. 8, no. 171, Sep. 2014. 